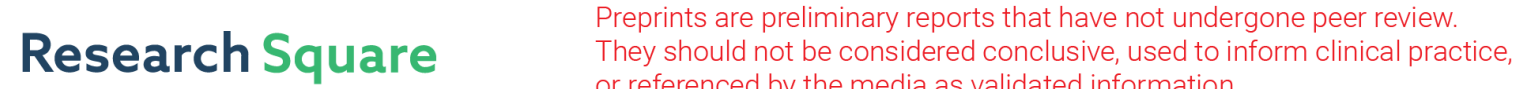 or referenced by the media as validated information. \\ Clinicopathological characteristics and outcomes of 23 patients with secretory carcinoma of major salivary glands
}

\section{Jingjing Sun}

the first affiliated hospital of Zhengzhou University

Sirui Liu

the first affiliated hospital of Zhengzhou University

\section{Kun Fu}

the first affiliated hospital of Zhengzhou University

\section{Ning Gao}

the first affiliated hospital of Zhengzhou University

Rui Li

the first affiliated hospital of Zhengzhou University

Wei He ( $\boldsymbol{\sigma}$ kqsjj@zzu.edu.cn )

The First Affiliated Hospital of Zhengzhou University

\section{Zhenjie Gao}

the first affiliated hospital of Zhengzhou University

\section{Research}

Keywords: Secretory carcinoma of salivary gland, Clinicopathological characteristics, Surgical procedure, Prognosis

Posted Date: February 26th, 2021

DOI: https://doi.org/10.21203/rs.3.rs-249091/v1

License: (c) (1) This work is licensed under a Creative Commons Attribution 4.0 International License. Read Full License 


\section{Abstract \\ Background}

Secretory carcinoma of salivary glands (SCSGs) are generally low-grade salivary gland carcinomas, and are characterized by morphological resemblance to mammary analogue secretory carcinoma and ETV6NTRK3 gene fusion. Several reports on histopathological features of SCSG have recently been published; however, little is known about the clinical characteristics of this new tumor, including its outcomes. The research is to investigate the clinicopathological characteristics of secretory carcinoma of major salivary glands and to analyze outcomes of these carcinomas as a reference for standardizing their diagnosis and treatment.

\section{Methods}

The cohort of this retrospective study comprised 23 patients treated for histopathologically-confirmed SCSG between January 2010 and December 2020. Their clinical characteristics and outcomes were retrieved from patient files.

\section{Results}

The 23 patients comprised 13 male and 10 female patients (male:female ratio 1.3:1). They were aged 10-69 years (median 45 years) and the average duration of disease was 2.44 years (0.25-20 years). Twenty-one patients (91.3\%) had SCSGs in the parotid gland and two (8.7\%) in the submandibular gland. All 23 patients had single nodules with diameters of $0.8-4.8 \mathrm{~cm}$ (average $2.6 \mathrm{~cm}$ ). Five patients had lymph node metastases and two had distant metastases. All tumors were pathologically diagnosed as SCSGs. Immunohistochemical staining was strongly positive for S-100, mammaglobin, CK7, and Gata3, and negative for Dog1, P63 and calponin. Ki-67 positivity ranged from 1-50\%. Fluorescence in situ hybridization data were available for 15 patients, in all of whom ETV6 gene rearrangement was confirmed. All patients had undergone oncological resections. Four had radioactive particles implanted postoperatively, one received chemotherapy, and seven underwent chemoradiotherapy. Six patients had regional recurrences, two distant metastases, and one had died at the time of last follow-up.

\section{Conclusions}

SCSGs are typically indolent with a low rate of locoregional recurrence and excellent survival. Their prognosis is correlated with clinical stage, pathological grade, and surgical procedures performed.

\section{Background}


The diagnosis of secretory carcinoma of salivary glands (SCSGs), a recently-described rare malignant tumor, was introduced by Skálová et al. in 2010 [1]. These tumors were originally classified as mammary analogue secretory carcinomas of salivary glands because their histomorphological and immunohistochemical features are similar to those of secretory carcinoma of the breast [2]. The updated World Health Organization (WHO) Classification of Head and Neck Tumors (4th edition, 2017) substituted secretory carcinoma for the previous mammary analogue secretory carcinoma designation [3].

These salivary gland tumors are characterized by the presence of a translocation $t(12 ; 15)(p 13 ; q 25)$, which leads to fusion of the ETV6 gene on chromosome 12 and the neurotrophic tropomyosin receptor kinase 3 (NTRK3) gene on chromosome 15 [4]. Before introduction of the diagnosis of SCSG, these tumors were mostly diagnosed as acinic cell carcinoma (ACC), mucoepidermoid carcinoma, adenocarcinoma, or not otherwise specified [5].

Several reports on histopathological features of SCSG have recently been published; however, little is known about the clinical characteristics of this new tumor, including its outcomes [6]. Here, we present a review of 23 patients with SCSG in major salivary glands who were treated in our department at the First Affiliated Hospital of ZhengZhou University, including detailed discussion of clinicopathological characteristics, outcomes of treatment, and prognosis.

\section{Methods}

This was a retrospective study and the protocol of the study was approved by the Medical Ethics Committee of our institution(Ethics Review Number: 2020-KY-230). All patients gave informed consent and signed informed consent forms. We retrospectively enrolled 23 patients with histopathologicallyconfirmed SCSG who had been treated between January 2010 and December 2020. The diagnoses of SCSG were blindly reviewed by three independent consultant pathologists, all experts in salivary gland pathology.

For conventional microscopy, formalin-fixed, paraffin-embedded tissues stained with hematoxylin and eosin were used. Selected immunohistochemical stains were used in all cases, including S-100, mammaglobin, CK7, Gata3, Dog1, P63, calponin, and Ki-67. Fluorescence in situ hybridization was performed to detect the ETV6 gene.

All patients' clinical data were analyzed for gender, age, size of tumor, symptoms and their duration, means of diagnosis, TNM stage, treatment administered, recurrences, and prognosis.

\section{Results}

Clinical characteristics

The 23 patients with SCSG comprised 13 male and 10 female patients (male:female ratio 1.3:1). The median age was 45 years (range 10-69 years) and the average duration of disease from initial 
symptoms to diagnosis was 2.44 years (range 3 months to 20 years). Twenty-one of the tumors (91.3\%) were located in the parotid gland (four left parotid, 17 right parotid) and two (8.7\%) in the submandibular gland. All 23 patients had single nodules presenting as painless masses of diameter $0.8-4.8 \mathrm{~cm}$ (average diameter $2.6 \mathrm{~cm})$. Six patients $(26.1 \%)$ had T1 tumors, 14 (60.9\%) T2 tumors, and three (13\%) T3 tumors. Five patients had presented with regional lymph node metastases and two had distant metastases at the time of diagnosis (Table 1).

\section{Table 1}

Patients' and tumor characteristics

\begin{tabular}{|ll|}
\hline Gender, $\mathbf{n}(\%)$ & \\
\hline Male & $13(56.5 \%)$ \\
\hline Female & $10(43.5 \%)$ \\
\hline Median age, in years[range] & $45(10-69)$ \\
\hline Tumor localization, $\mathrm{n}(\%)$ & \\
\hline Major salivary glands & \\
\hline -parotid (left, right) & $21(91.3 \%$, left 4, right 17$)$ \\
\hline -submandibular gland & $2(8.7 \%$, left 1, right 1$)$ \\
\hline Tumor characteristics & \\
\hline size (cm) & $2.6(0.8-4.8)$ \\
\hline texture & firm \\
\hline boundary & well-demarcated \\
\hline fixation & elastic and mobile \\
\hline Signs and symptoms & painless and no facial paralysis \\
\hline TNM stage, $n$ & \\
\hline T1/T2/T3/T4/Tx & $6 / 14 / 3 / 0 / 0$ \\
\hline N0/N1/N2/N3 & $18 / 1 / 4 / 0$ \\
\hline M0/M1 & $21 / 2$ \\
\hline *Based on 23 patients & \\
\hline
\end{tabular}

All 23 patients had undergone surgery as their primary treatment. The affected salivary gland was resected in 14 patients. Seven patients had undergone local excision. The precise extent of surgery could not be determined for the remaining two patients. None of the first surgeries included a neck dissection. 
Details of treatment administered and outcomes are summarized in Table 2. One of the 11 patients who had been treated with surgery only had a local recurrence 42 months after the primary surgery. Four patients had undergone post-operative implantation of $\mathrm{I}^{125}$ radioactive particles under MRI navigation (average of 35 particles); a typical case is shown in Fig. 1. None of these patients had local recurrences. The one patient who received chemotherapy had a local recurrence and four of the seven who had undergone chemoradiotherapy had local recurrences. Five patients developed lymphatic metastases (21.7\%), two developed distant metastases (8.7\%), and one patient died during follow-up (4.3\%).

\section{Table 2}

Outcomes of 23 patients

With SCSG treated by different therapies

\begin{tabular}{|c|c|c|c|c|c|}
\hline Treatments & cases & recurrence & $\begin{array}{l}\text { Lymphatic } \\
\text { metastasis }\end{array}$ & $\begin{array}{l}\text { Distant } \\
\text { metastasis }\end{array}$ & Death \\
\hline Operation only & 11 & 1 & 0 & 0 & 0 \\
\hline Operation $+I^{125}$ & 4 & 0 & 1 & 0 & 0 \\
\hline $\begin{array}{l}\text { Operation + } \\
\text { chemotherapy }\end{array}$ & 1 & 1 & 1 & 0 & 0 \\
\hline $\begin{array}{l}\text { Operation + } \\
\text { chemoradiotherapy }\end{array}$ & 7 & 4 & 3 & 2 & 1 \\
\hline Total & 23 & 6 & 5 & 2 & 1 \\
\hline
\end{tabular}

Imaging findings

On color doppler ultrasound, the tumors appeared as well-demarcated, heterogeneous, hypoechoic masses with regular morphology. Most of them were solid, some being a combination of cystic and solid. Punctate blood flow signals were seen within the lesions; these can easily prompt misdiagnosis as pleomorphic adenoma. Typical ultrasonic characteristics are shown in Fig. 2F.

With computed tomography (CT), the lesions appeared oval or lobulated, with clear boundaries, regular edges, and uneven density. Some had low-density cystic areas and foci of calcification(Fig. 2A-C). The average $\mathrm{HU}$ was $42(41.69 \pm 16.5)$ with plain scanning and $76(76.1 \pm 24.7)$ with enhanced scanning (Fig. 2D-E). The degree of enhancement varied, mostly being uneven and marked (>40 HU). However, a few tumors showed mild enhancement $(10-20 \mathrm{HU})$ or no obvious enhancement $(<10 \mathrm{HU})$. Most of the recurrences showed marked enhancement. Slightly enlarged lymph nodes were found in the ipsilateral submaxillary region and carotid sheath, potentially resulting in the misdiagnosis of pleomorphic adenoma. 
We found that SGSCs consistently had the following characteristics on magnetic resonance imaging (MRI): (1) a tendency to be roundish in shape, with clear boundaries; and (2) most T1 or T2 images showed long signals or mixed long and short signals. Fat suppression images showed high signals. On diffusion weighted imaging, there was little high b value diffusion, with high signals and uneven enhancement ranging from slight to significant. As with the CT findings, slightly enlarged lymph nodes were found in the ipsilateral submaxillary region and carotid sheath, as shown in Fig. 3A-C.

Moreover, two patients in our study group had undergone magnetic resonance hydrography (MRH), which showed that in both cases the lesions were located in the posterior lower pole of the parotid gland, more than $3 \mathrm{~mm}$ away from the main duct, which appeared normal. Regional parotid gland resection was therefore performed to preserve the secretory function of the parotid duct and part of that gland, as shown in Fig. 3D-F.

Pathological characteristics

Grossly, the tumors were isolated solid nodules with light-tan or grayish cut surfaces (Fig. 4A). Their texture was hard, they had clear boundaries, and their average diameter was $2.6 \mathrm{~cm}(0.8-4.8 \mathrm{~cm})$. A few of them harbored cysts containing yellowish-white fluid (Fig. 4B).

The tumors exhibited a lobulated growth pattern with fibrous septa and were composed of microcystic/solid, tubular, follicular and papillary cystic structures with distinctive luminal secretions (Figs. 4C-F). At high magnification, the tumor cells were round or oval with little atypia. They had eosinophilic granular or vacuolated cytoplasm with small, uniform nuclei (Fig. 4C, green box).

In our study group, immunohistochemical staining showed strong positivity for S-100 (Fig. 5A), mammaglobin (Fig. 5B), CK7 (Fig. 5C), and Gata3 (Fig. 5D), whereas staining was negative for calponin (Fig. 5E), P63 (Fig. 5F), and Dog1 (Fig. 5G). Ki-67 positivity (Fig. 5H) ranged from $1-50 \%$, being $\leq 20 \%$ in 19 patients and $\leq 10 \%$ in 14 patients. Fluorescence in situ hybridization data were available for 15 patients (Fig. 6). The tumors of 15 patients that had not been definitely diagnosed by immunohistochemistry were diagnosed on the basis of ETV6 gene rearrangement, this being found in all 15 patients.

\section{Discussion}

SCSGs are generally low-grade salivary gland carcinomas, and are characterized by morphological resemblance to mammary analogue secretory carcinoma and ETV6-NTRK3 gene fusion $[2,7]$. In 2017, SCSGs were added to the WHO classification of head and neck tumors [3].

The age of onset of SCSGs reportedly varies widely. These tumors usually develop in adults, but can occur in children and adolescents; there is no significant sex difference [8]. In the present group, the median age was 45 years (range: 10-69 years) (male:female ratio 1.3:1). The parotid gland is the most common site of SCSGs; small salivary glands such as the buccal, upper lip, and palatal glands can also 
be involved $[9,10,11]$. The tumors of 21 of the 23 patients in the present study were located in the parotid gland, 17 being in the right parotid and four in the left parotid gland, differing slightly from that previously reported [12]. SCSGs most commonly present as painless, slow-growing, well-circumscribed masses, and can be misdiagnosed as pleomorphic adenoma or acinar cell carcinoma [13]. Consistent with previous reports, most patients presented after finding a painless mass, with only a few recurrences being sensitive to touch. In the present study, no specific pre-operative ultrasonography, CT or MRI findings were identified. The tumors presented as well-demarcated masses of uneven density, sometimes with cystic changes, that could easily be misdiagnosed as pleomorphic adenomas. There were only three patients with T3 tumors in our cohort, the others being T1 or T2 tumors. However, T4 tumors have been reported by others [12].

Before their recognition, SCSGs were frequently classified as ACCs because of the nearly identical histological growth patterns of these tumor types [14]. There are no significant clinical differences between SCSGs and ACCs. However, these tumors may show considerable morphological differences histopathologically. Unlike ACCs, SCSGs have no secretory zymogen cytoplasmic granules that show true positive periodic acid-Schiff reactions [15]. The nuclei of SCSGs have prominent pseudo-inclusion bodies and SCSGs do not have the other cell types, such as serous, intermediate, and clear cells, that are seen in ACCs [16]. As to immunophenotypes, SCSGs can express various markers of breast secretory carcinomas, including mammaglobin, S-100 protein, STAT5A, MUC1, MUC4, GCDFP-15, as well as CK7, CK8, CK18, CK19, and epithelial cell membrane proteins [17]. However, they characteristically do not express DOG-1, estrogen, androgen, progesterone receptors, or HER2 [18]. In contrast with SCSGs, ACCs do not express mammaglobin, or express it weakly locally, whereas they express D0G-1 diffusely [18]. Khalele et al. showed that SCSG can be diagnosed when a salivary gland tumor expresses mammaglobin and S-100 protein strongly, and does not express DOG-1 [19]. It has also been reported that if mammaglobin and S-100 are strongly positive, SCSG can be diagnosed without resorting to molecular biological methods [20]. In particular, mammaglobin has become a very important tumor marker for diagnosing SCSGs.

Similarly to secretory carcinomas of the breast, SCSGs have been shown to have the translocation $\mathrm{t}(12$; 15) (p13; q25), which results in fusion of the ETV6 gene on chromosome 12 with the NTRK3 gene on chromosome 15 [4]. ETV6-NTRK3 gene fusion has not been found in other types of salivary gland tumor. However, it has been found in congenital fibrosarcoma, mesodermal renal tumor, and acute myeloid leukemia $[21,22]$. Recent findings have expanded the molecular profile of SCSG to include multiple novel ETV6 fusion partners, including ETV6-RET and EGFR-SEPT14 [23]. Rupture of ETV6 and its fusion with NTRK3 may lead to production of functional tyrosine kinases or affect the signaling pathways of some transcriptional activators, leading to strong expression of mammaglobin and S100, but not of DOG-1 [24]. It is therefore considered that detection of ETV6 gene rearrangement is the most specific method, and therefore the gold standard, for diagnosing SCSGs. Additionally, gene therapy may offer new hope to patients with this tumor in the future [25]. In our study, the tumors of 15 patients that had not been definitely diagnosed by immunohistochemistry were diagnosed on the basis of ETV6 gene rearrangement, this being found in all 15 cases. Chiosea reviewed 81 cases of ACC for ETV6 fluorescence 
by in situ hybridization and found that all classical ACCs had intact ETV6, whereas 10 of 17 zymogen granule-poor ACCs showed ETV6 translocation; these were accordingly reclassified as mammary analog secretory carcinomas [26]. Other differential diagnoses include adenoid cystic carcinoma, mucoepidermoid carcinoma, and salivary ductal carcinoma, all of which can identified by immunohistochemistry and ETV6 gene detection. Before immunohistochemistry and ETV6 gene detection had resulted in the correct diagnoses of SCSG being made, four of the present 23 patients' tumors were misdiagnosed as acinar cell carcinomas, three as mucoepidermoid carcinomas, two as adenoid cystic carcinomas, one as low-grade ductal carcinoma, and one as squamous cell carcinoma. Thus, the rate of misdiagnosis with routine pathology only was $47.8 \%$.

Because SCSGs behave similarly to ACCs, the treatment of SCSGs is mainly surgical. However, there is currently no consensus on optimal treatment of these tumors. Some researchers believe that SCSGs are indolent tumors that rarely produce distant metastases and that surgery alone is sufficient [27]. There have also been reports that surgery combined with postoperative radiotherapy is more effective [28]. In the present study, 11 patients were treated with surgery alone, one of whom had a recurrence postoperatively. Radioactive $\mathrm{I}^{125}$ implantation was performed under MRI navigation in four patients, none of whom developed recurrences. In addition, one patient received postoperative chemotherapy and later had a recurrence. Four of seven patients who received postoperative chemoradiotherapy had recurrences. The overall recurrence rate was $26.1 \%$.

Interestingly, six of the patients with recurrences had undergone their first surgeries in local primary hospitals. Their recurrences were considered to have resulted from incomplete resection by the first surgery. There are currently few reports of different surgical procedures for SCSGs. Some studies advocate simultaneous total resection of the affected lobe of the parotid gland and cervical lymph node dissection [29]. Chiosea et al. reported a higher incidence of lymph node metastases from SCSGs than from ACCs (33\% vs. $8 \%$ ); despite this, cervical dissection is still rarely performed [30]. Five of the present 23 patients developed cervical lymphatic metastases and two developed distant metastases (both to the lung) after having local recurrences. None of the patients who had undergone excision of the tumor and superficial lobe of the parotid gland had recurrences or distant metastases. All six patients with local recurrences had only undergone tumor enucleation or partial parotid gland resection, which suggests that preoperative determination of the size and extent of the tumor is vital to the success of surgery.

It has recently been shown that MRH of salivary gland ducts can clearly establish the three-dimensional relationship between the tumor and parotid duct and detect dilation, stenosis, displacement, and destruction of main ducts and branch ducts, potentially guiding preoperative planning [31]. If the tumor has invaded the main duct, retaining the gland is pointless and superficial or total parotid gland resection should be performed where possible. If the tumor is sufficiently distant from the main duct, regional parotid gland resection with preservation of part of the gland can be considered. Thus, detailed MRHs can accurately guide the planning of parotid gland preservation surgery, potentially enabling retention of some functional gland while completely resecting the tumor. In the present study, two patients had undergone preoperative magnetic resonance hydrography and regional resection of the parotid gland 
without any other postoperative treatment. Thus far, they remain well, their average follow-up time being 26 months.

The prognosis of SCSG is related not only to the first operative procedure, but also to age, clinical stage, and Ki-67 proliferation index. Although six of 23 patients in this study had local recurrences (mean age 53 years) and one patient died, the overall prognosis was good, which is consistent with reported results [32]. Additionally, the prognosis is age-dependent. Children and young patients have better prognoses, adult patients having more aggressive tumors. Older patients have the most aggressive tumors and highest recurrence rate [33]. This may be related to differences in pathological features at different ages and the characteristically poor immune function of older patients. Furthermore, previous studies have shown that high clinical stage and high grade are the main adverse prognostic factors, lymph node involvement and extra-parenchymal glandular invasion being associated with a greater risk of local recurrence and metastasis [34]. In the present study, five patients developed lymph node metastases, all of whom underwent therapeutic neck dissection and postoperative radiotherapy and/or chemotherapy. However, they all had postoperative recurrences, with pulmonary metastases in two of them, one of whom died. Additionally, the tumor of the patient who died had a Ki-67 proliferation index of $50 \%+$ and had recurred three times. This is consistent with reports that patients with a Ki-67 proliferation index of more than $10 \%$ have poor prognoses, suggesting that the $\mathrm{Ki}-67$ proliferation index affects the prognosis of secretory carcinomas [35].

\section{Conclusions}

In summary, SCSGs are a rare type of low-grade malignant salivary gland tumor. They commonly occur in the parotid gland, rarely invade surrounding tissues, and have a good prognosis. Histomorphological and immunohistochemical characteristics are the key to distinguishing SCSGs from other salivary gland tumors, detection of ETV6 translocation being the gold standard for their diagnosis. Surgical resection is the main treatment and the thoroughness of the first operation is the major determinant of prognosis. Considering the low rate of cervical lymphatic metastasis, functional excision of SCSGs with facial nerve preservation is generally performed, depending on preoperative MRH findings concerning salivary gland duct status. When considered indicated, postoperative $\mathrm{I}^{125}$ implantation or local radiotherapy can be performed to reduce the recurrence rate. If distant metastases occur, neck dissection and postoperative treatment are necessary. Because SCSG is a relatively newly-described tumor type, few clinical findings have been reported. There were only a small number of patients in our study cohort. Thus, definitively ascertaining the optimal treatment and outcomes of SCSG still requires large studies and long-term follow-up.

\section{List Of Abbreviations}

SCSG secretory carcinoma of salivary gland

SCSGs secretory carcinoma of salivary glands 
NTRK3 neurotrophic tropomyosin receptor kinase 3

ACC acinic cell carcinoma

CT computed tomography

MRI magnetic resonance imaging

MRH magnetic resonance hydrography

\section{Declarations}

\section{Ethics approval and consent to participate}

This was a retrospective study and the protocol of the study was approved by the Medical Ethics Committee of our institution(Ethics Review Number: 2020-KY-230). All patients gave informed consent and signed informed consent forms.

\section{Consent for publication}

All participants provided informed written consent.

\section{Availability of data and materials}

The datasets analysed during this study are included in this published article.

\section{Competing interests}

The authors declare that have no competing interests.

\section{Funding}

This work was supported by Natural Science Foundation of China (81901039).

\section{Authors' contributions}

JJ. S contributed to the manuscript preparation and performed significantly the data analyses and wrote the manuscript; SR. L, K. F, N. G and R. L helped perform the analysis with constructive discussions; W. H and ZJ. G contributed to the conception of the study and manuscript preparation.

\section{Acknowledgment}

Not applicable.

\section{References}


1. Skálová A, et al. Mammary analogue secretory carcinoma of salivary glands, containing the ETV6NTRK3 fusion gene: a hitherto undescribed salivary gland tumor entity. Am J Surg Pathol. 2010;34:599-608.

2. Hindocha $\mathrm{N}$, Wilson $\mathrm{MH}$, Pring $\mathrm{M}$, et al. Mammary Analogue Secretory Carcinoma of the Salivary Glands: A Diagnostic Dilemma. Br J Oral Maxillofac Surg. 2017;55:290-92.

3. Bissinger O, Gotz C, Kolk A, et al. Mammary Analogue Secretory Carcinoma of Salivary Glands: Diagnostic Pitfall with Distinct Immunohistochemical Profile and Molecular Features. Rare Tumors. 2017;9:7162.

4. Naous R, Zhang S, Valente A, et al. Utility of Immunohistochemistry and Etv6 (12p13) Gene Rearrangement in Identifying Secretory Carcinoma of Salivary Gland among Previously Diagnosed Cases of Acinic Cell Carcinoma. Patholog Res Int. 2017; 2017:1497023.

5. Hamamoto $Y$, Harada $H$, Kohara $M$, et al. Usefulness of Immunohistochemistry to Distinguish between Secretory Carcinoma and Acinic Cell Carcinoma in the Salivary Gland. Med Mol Morphol. 2020. doi:10.1007/s00795-020-00256-4.

6. Alves LDB, Melo ACD, Farinha TA, et al. A Systematic Review of Secretory Carcinoma of the Salivary Gland: Where Are We? Oral Surg Oral Med Oral Pathol Oral Radiol. 2020. doi:10.1016/j.0000.2020.04.007.

7. Parekh V, Stevens TM. Mammary Analogue Secretory Carcinoma. Arch Pathol Lab Med. 2016;140:997-1001.

8. Lee DH, Kim JH, Yoon TM, et al. Outcomes of Treatment of Mammary Analogue Secretory Carcinoma of the Parotid Gland. Br J Oral Maxillofac Surg. 2020;58:158-62.

9. Zardawi IM, Hook P. Mammary Analogue Secretory Carcinoma of Minor Salivary Glands Pathology. 2014;46:667-9.

10. Kratochvil FJ, Stewart JC, Moore SR. Mammary Analog Secretory Carcinoma of Salivary Glands: A Report of 2 Cases in the Lips. Oral Surg Oral Med Oral Pathol Oral Radiol. 2012;114:630-5.

11. Takano H, Fukuda M, Hatakeyama S, et al. A Case of Secretory Carcinoma of the Minor Salivary Gland in the Buccal Mucosa. Journal of Oral Maxillofacial Surgery Medicine Pathology. 2020. doi.org/10.1016/j.ajoms.2020.08.009.

12. Boon E, Valstar MH, vanderGraaf WTA, et al. Clinicopathological Characteristics and Outcome of 31 Patients with Etv6-Ntrk3 Fusion Gene Confirmed (Mammary Analogue) Secretory Carcinoma of Salivary Glands. Oral Oncol. 2018;82:29-33.

13. Bin X, Nora K. Evolving Concepts and New Entities in the 2017 Who Classification of Salivary Gland Tumors. Diagnostic Histopathology. 2018;24:172-79.

14. Guilmette J, Nielsen GP, Faquin WC, et al. Ultrastructural Characterization of Mammary Analogue Secretory Carcinoma of the Salivary Glands: A Distinct Entity from Acinic Cell Carcinoma? Head Neck Pathol. 2017;11:419-26.

15. Speight PM, Barrett AW. Salivary Gland Tumours: Diagnostic Challenges and an Update on the Latest Who Classification. Diagnostic Histopathology. 2020;26:147-58. 
16. Mossinelli C, Pigni C, Sovardi F, et al. Synchronous Parotid (Mammary Analog) Secretory Carcinoma and Acinic Cell Carcinoma: Report of a Case. Head Neck Pathol. 2018;13:686-91.

17. Projetti F, Lacroix-Triki M, Serrano E, et al. A comparative immunohistochemistry study of diagnostic tools in salivary gland tumors:usefulness of mammaglobin,gross cystic disease fluid protein 15 ,and p63 cytoplasmic staining for the diagnosis of mammary anolog secretory carcinoma? J Oral Pathol Med. 2015;44:244-51.

18. Krings G, Joseph NM, Bean GR, et al. Genomic Profiling of Breast Secretory Carcinomas Reveals Distinct Genetics from Other Breast Cancers and Similarity to Mammary Analog Secretory Carcinomas. Mod Pathol. 2017;30:1086-99.

19. Khalele BA. Systematic Review of Mammary Analog Secretory Carcinoma of Salivary Glands at 7 Years after Description. Head Neck. 2017;39:1243-48.

20. Griffith C, Seethala R, Chiosea SI. Mammary Analogue Secretory Carcinoma: A New Twist to the Diagnostic Dilemma of Zymogen Granule Poor Acinic Cell Carcinoma. Virchows Arch. 2011;459:117-8.

21. Majewska H, Skálová A, Stodulski $D$, et al. Mammary analogue secretory carcinoma of salivary glands: a new entity associated with ETV6 gene rearrangement.Virchows Arch. 2015; 466:245-54.

22. Baghai F, Yazdani F, Etebarian A, et al. Clinicopathologic and Molecular Characterization of Mammary Analogue Secretory Carcinoma of Salivary Gland Origin. Pathol Res Pract. 2017;213:1112-18.

23. Black M, Liu CZ, Onozato M, et al. Concurrent Identification of Novel Egfr-Sept14 Fusion and Etv6-Ret Fusion in Secretory Carcinoma of the Salivary Gland. Head Neck Pathol. 2020;14:817-21.

24. Connor A, Perez-Ordonez B, Shago M, et al. Mammary Analog Secretory Carcinoma of Salivary Gland Origin with the Etv 6 Gene Rearrangement by Fish: Expanded Morphologic and Immunohistochemical Spectrum of a Recently Described Entity. Am J Surg Pathol. 2012;36:27-34.

25. Xu B, Rasheed HA, et al. Pan-Trk Immunohistochemistry Is a Sensitive and Specific Ancillary Tool for Diagnosing Secretory Carcinoma of the Salivary Gland and Detecting Etv6-Ntrk3 Fusion. Histopathology. 2020;76:375-82.

26. Chiosea SI, et al. The Profile of Acinic Cell Carcinoma After Recognition of Mammary Analog Secretory Carcinoma. the American journal of surgical pathology. 2012;36:343-50.

27. Sun L, Thorson T, Zhu R, Huo J, et al. A Case Report of Parotid Mammary Analogue Secretory Carcinoma and Reviews. Int J Surg Case Rep. 2019;55:88-91.

28. Tokuzen N, Goda H. and Nakashiro K. Locally Advanced Mammary Analogue Secretory Carcinoma of the Parotid Gland. Int J Oral Maxillofac Surg. 2019;48:865-68.

29. Lee DH, Kim JH, Yoon TM, et al. Outcomes of Treatment of Mammary Analogue Secretory Carcinoma of the Parotid Gland. Br J Oral Maxillofac Surg. 2020;58:158-62.

30. Williams L, Chiosea SI. Mammary Analogue Secretory Carcinoma Mimicking Salivary Adenoma. Head Neck Pathol. 2013;7:316-9. 
31. Bissinger O, Gotz C, Kolk A, et al. Mammary Analogue Secretory Carcinoma of Salivary Glands: Diagnostic Pitfall with Distinct Immunohistochemical Profile and Molecular Features. Rare Tumors. 2017;9:7162.

32. Boliere C, Murphy J, Qaisi M, et al. Mammary Analogue Secretory Carcinoma of the Palate: Case Report and Review of the Literature. Case Rep Dent. 2019;7416302.

33. Chen YC, Hsu HT, Lin YH, et al. Mammary Analog Secretory Carcinoma of the Parotid Gland: A Case of a 12-Year-Old Taiwanese Girl. International Journal of Pediatric Otorhinolaryngology Case Reports. 2018;20:14-7.

34. Cipriani NA, Blair EA, Finkle J, et al. Salivary Gland Secretory Carcinoma with High-Grade Transformation, Cdkn2a/B Loss, Distant Metastasis, and Lack of Sustained Response to Crizotinib. Int J Surg Pathol. 2017;25:613-18.

35. Chiosea SI, Griffith C, Assaad A, Seethala RR. Clinicopathological characterization of mammary analogue secretory carcinoma of salivary glands. Histopathology. 2012;61:387-94.

\section{Figures}



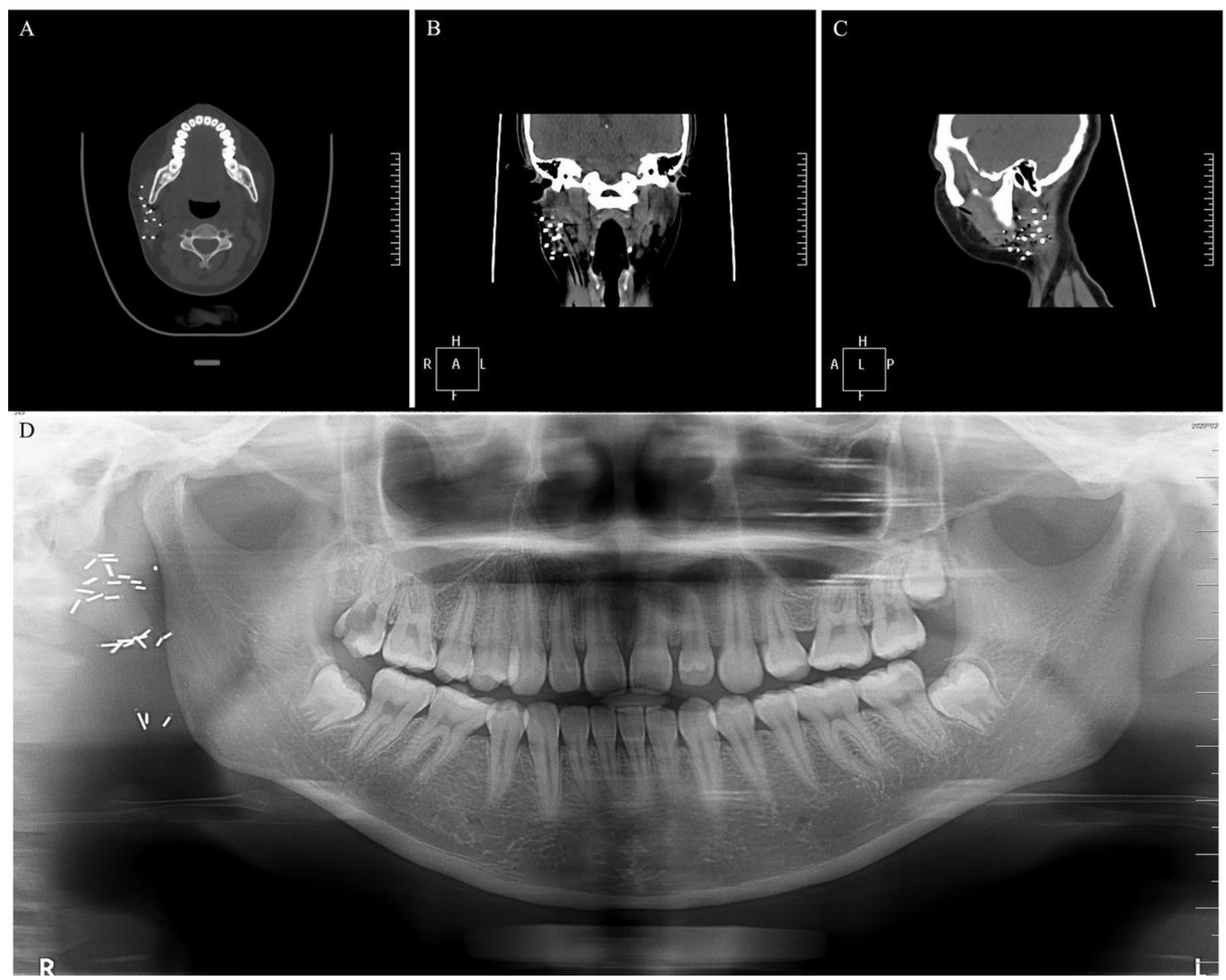

\section{Figure 1}

A typical post-operative implantation of 1125 radioactive particles (A) horizontal plane (B) coronal plane (C) sagittal plane (D) panoramic radiography 


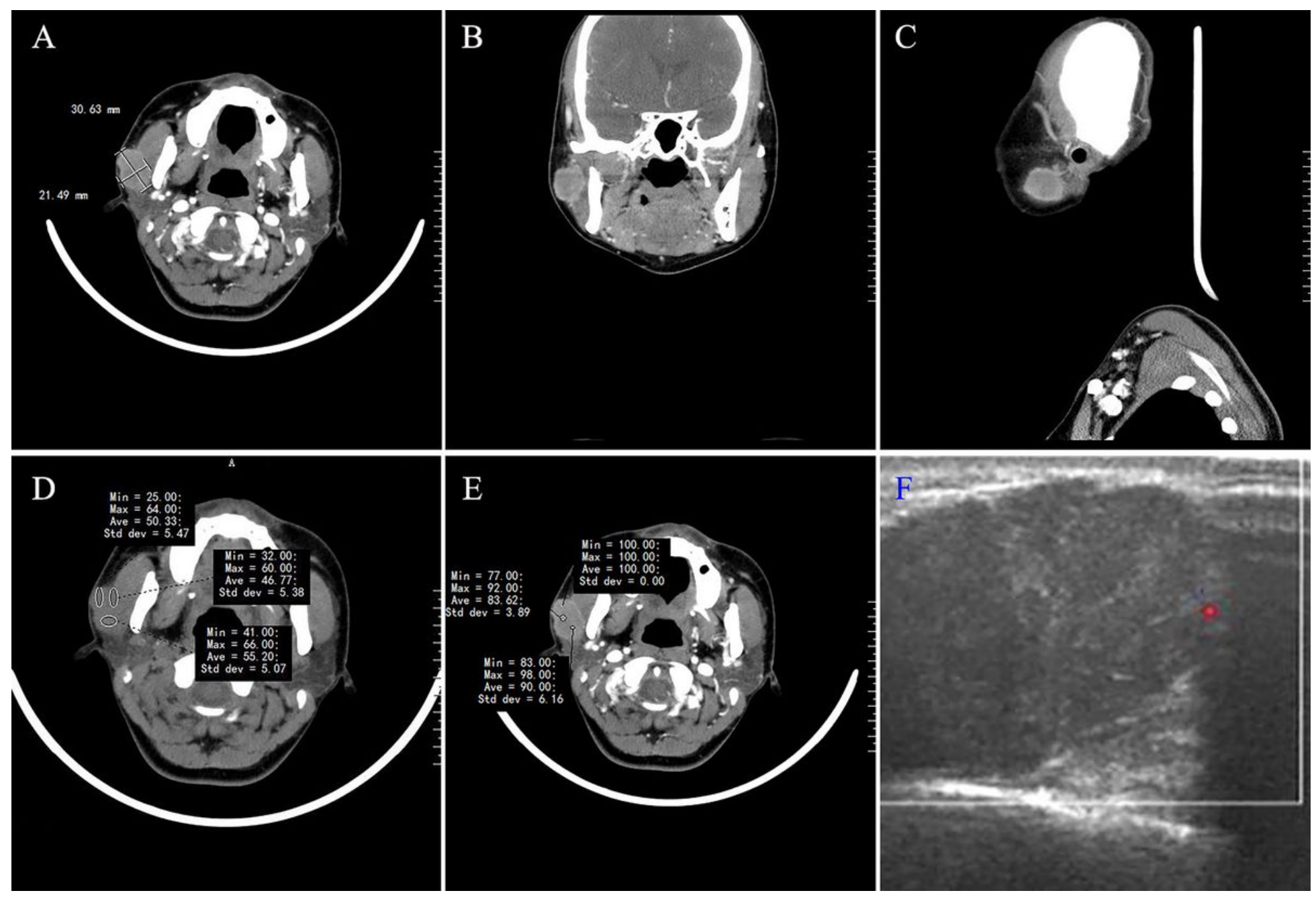

Figure 2

Color doppler ultrasound and computed tomography (CT) imaging findings of typical SCSG With CT, the lesions appeared oval or lobulated, with clear boundaries, regular edges, and uneven density (A) horizontal plane (B) coronal plane (C) sagittal plane; (D) average HU was $42(41.69 \pm 16.5)$ with plain scanning and $(E) 76(76.1 \pm 24.7)$ with enhanced scanning; $(F)$ typical ultrasonic characteristics 

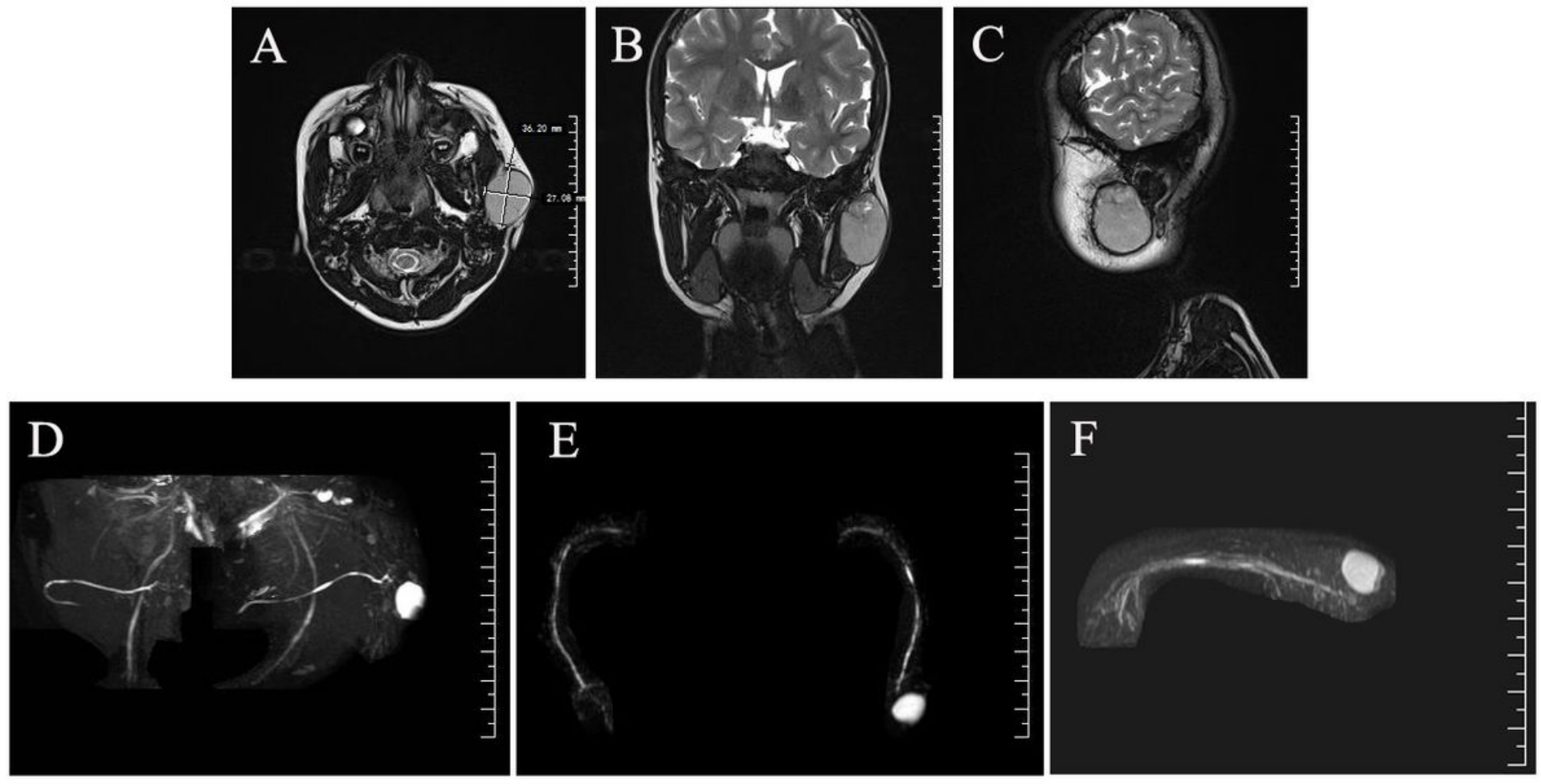

\section{Figure 3}

On MRI, tumor tend to be roundish in shape, with clear boundaries, and T2 images showed long signals or mixed long and short signals, (A) horizontal plane (B) coronal plane (C) sagittal plane; MRH showed the lesions were located in the posterior lower pole of the parotid gland (D) coronal plane (E) horizontal plane (F) sagittal plane 

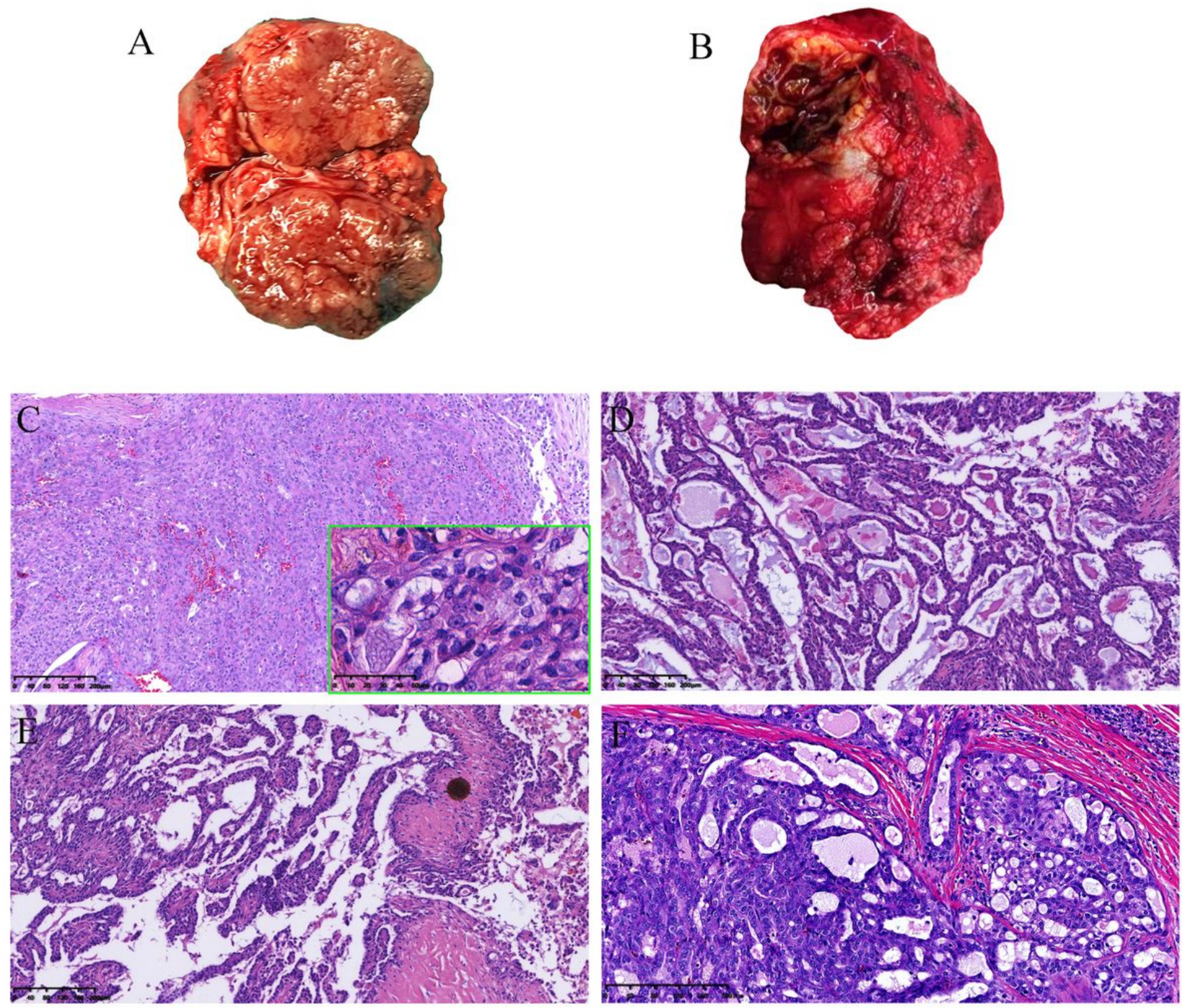

Figure 4

(A) The tumor was isolated solid nodule with light-tan or grayish cut surface; (B) A tumor harbored cysts containing yellowish-white fluid; (C) solid structures (D) microcystic structures (E) follicular and papillsry cystic structures (F) tubular structures; At high magnification, the tumor cells were round or oval with little atypia. They had eosinophilic granular or vacuolated cytoplasm with small, uniform nuclei (green box). 

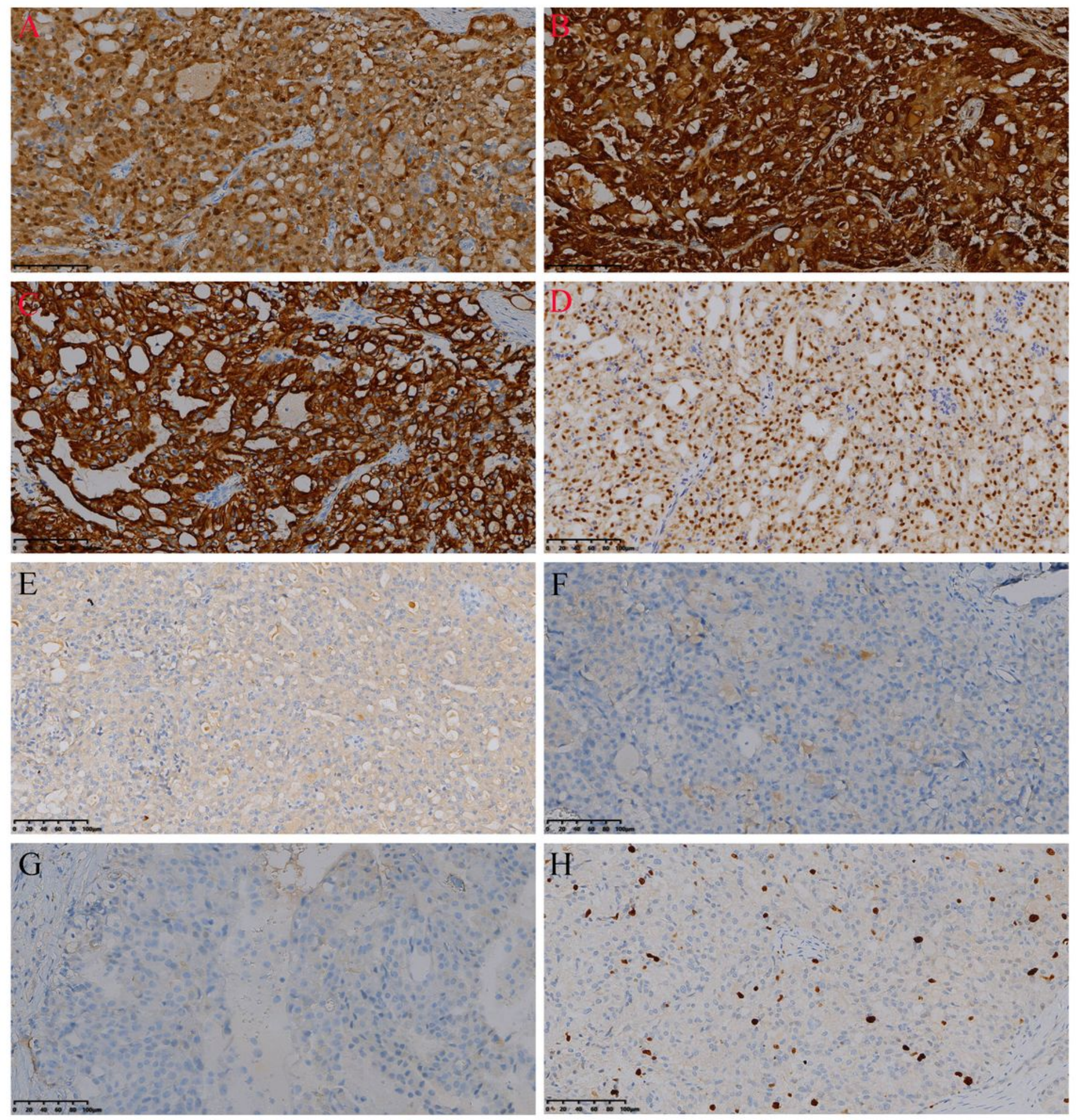

\section{Figure 5}

Immunohistochemical staining showed strong positivity for S-100 (A), mammaglobin (B), CK7 (C), and Gata3 (D), whereas staining was negative for calponin (E), P63 (F), and Dog1 (G). (H) Ki-67(+) was 5\% 


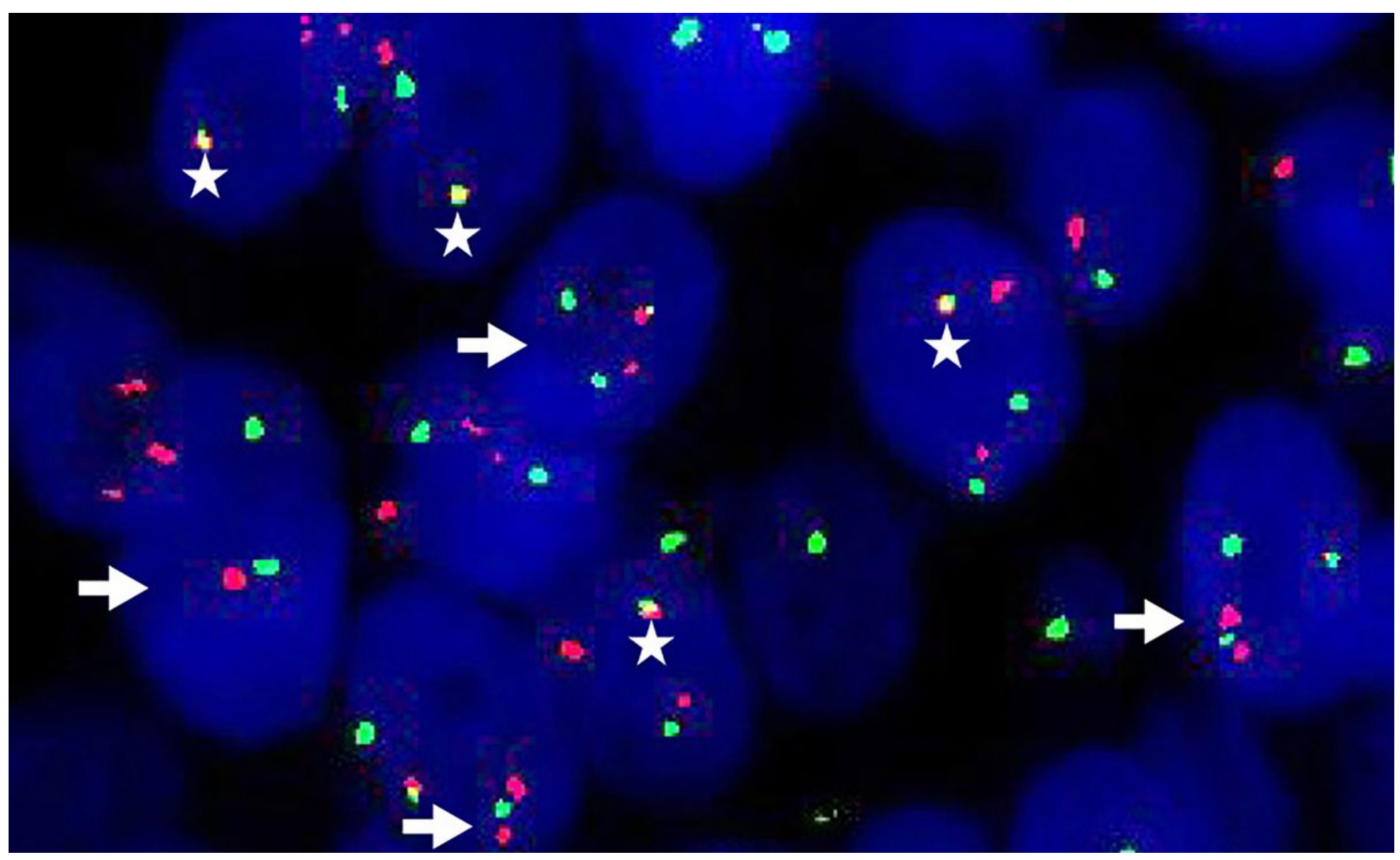

\section{Figure 6}

ETV6 gene rearrangement was confirmed by Fluorescence in situ hybridization as tumor cells having split signals (arrows) adjacent to NTRK3 (asterisks). 